\title{
Jejunal Diverticular Perforation due to Enterolith
}

\author{
Ronaldo Nonose ${ }^{a} \quad J u l i a n a$ Santos Valenciano ${ }^{a}$ \\ Jacintho Soares de Souza Lima ${ }^{a}$ Enzo Fabrício Nascimento ${ }^{a}$ \\ Camila Morais Gonçalves Silva ${ }^{b} \quad$ Carlos Augusto Real Martinez ${ }^{a}$ \\ aDepartment of General Surgery, São Francisco University Hospital, and

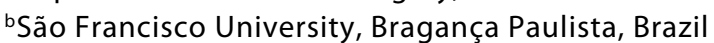

\section{Key Words}

Diverticulum $\cdot$ Intestines $\cdot$ Lithiasis $\cdot$ Intestinal perforation $\cdot$ Diverticulitis

\begin{abstract}
Jejunal diverticulosis is a rare entity with variable clinical and anatomical presentations. Although there is no consensus on the management of asymptomatic jejunal diverticular disease, some complications are potentially life-threatening and require early surgical treatment. Small bowel perforation secondary to jejunal diverticulitis by enteroliths is rare. The aim of this study was to report a case of small intestinal perforation caused by a large jejunal enterolith. An 86-year-old woman was admitted with signs of diffuse peritonitis. After initial fluid recovery the patient underwent emergency laparotomy. The surgery showed that she had small bowel diverticular disease, mainly localized in the proximal jejunum. The peritonitis was due to intestinal perforation caused by an enterolith $12 \mathrm{~cm}$ in length, localized inside one of these diverticula. The intestinal segment containing the perforated diverticulum with the enterolith was removed and an end-to-end anastomosis was done to reconstruct the intestinal transit. The patient recovered well and was discharged from hospital on the 5th postoperative day. There were no signs of abdominal pain 1 year after the surgical procedure. Although jejunal diverticular disease with its complications, such as formation of enteroliths, is difficult to suspect in patients with peritonitis, it should be considered as a possible source of abdominal infection in the elderly patient when more common diagnoses have been excluded.
\end{abstract}




\section{Introduction}

Jejunal diverticulosis (JD) is an uncommon disease and usually asymptomatic [1]. The literature estimates that JD has an incidence of $0.06-1.3 \%$ although the prevalence seems to increase with age, making it a topic of discussion in older patients presenting with abdominal pain [2]. The highest incidence of JD occurs during the sixth and seventh decades of life, and the disease is thought to be more common in males. Whereas it is frequently seen in the duodenum, jejunal and ileal locations are very rare. Nearly $80 \%$ of small bowel diverticula occur in the jejunum, approximately $15 \%$ in the ileum, and $5 \%$ in both [3].

More commonly, JD is diagnosed as an incidental finding of computed tomography (CT) imaging, small bowel barium radiographic series, or during surgery [3, 4]. It presents as pulsion diverticula thought to be the result of intestinal dyskinesia leading to high intraluminal pressure [3]. This high intraluminal pressure results in herniation of mucosa and submucosa through the weakest site of the muscularis, which is where blood vessels penetrate into the bowel wall. This explains the common location of JD at the mesenteric side of the bowel $[3,5]$.

The majority of patients with JD have no symptoms. As a result, preoperative identification of the disorder can be quite difficult [3]. Some patients have a history of chronic symptoms such as vague abdominal discomfort, fullness, or recurrent central and upper abdominal cramping pain caused by pseudoobstruction [6]. Anemia due to iron deficiency and megaloblastic anemia have often been reported and commonly attributed to malabsorption, steatorrhea, and B12 vitamin deficiency [1]. Malabsorption could be explained by the nonsynchronous peristaltic movement of the bowel, the dilation of the diverticula, the stasis of the intestinal content and the bacterial overgrowth. However, patients with JD can present with a number of acute and emergent complications such as massive gastrointestinal bleeding, intestinal obstruction or perforation with a high mortality rate $[1,4,7]$. Obstruction may also occur secondary to adhesions, bowel volvulus, or due to migration and impaction of enteroliths. Enteroliths may form inside the JD and consist of choleic acid, either de novo or around a bezoar $[1,8,9]$. The acidic environment within diverticula is ideal for aiding the metabolism of bile salts to choleic acid, hence enterolith obstruction [9].

Cases of JD complicated by enteroliths are indeed rare, but worth mentioning as they can produce an enterolith ileus or lead to perforation if the enterolith becomes impacted [2]. To the best of our knowledge only 39 cases of small bowel complication due to jejunal enterolithiasis have been reported in the literature [8]. The purpose of the present report is to present a case of small bowel obstruction and perforation due to an enterolith formed inside a JD that was successfully treated by intestinal resection.

\section{Case Report}

An 86-year-old woman presented to the emergency department with a 6-hour history of generalized abdominal pain mainly located in the periumbilical region, without fever, with five episodes of vomiting and stopped passage of gas and feces. The patient denied previous alterations of her bowel habits or rectal bleeding. She complained of previous occasional episodes of abdominal colicky pain with lesser 
intensity and had noted a weight loss of $4 \mathrm{~kg}$ in the last 4 months. She had a past medical history of hypertension with regular use of furosemide, methyldopa and angiotensin receptor antagonist. On physical examination the vital signs showed a temperature of $36^{\circ} \mathrm{C}$, a heart rate of $115 \mathrm{bpm}$, and a blood pressure of 100/80 mm Hg. Physical examination of the abdomen revealed generalized abdominal tenderness and signs of diffuse peritonitis. There was presence of a reducible umbilical hernia without signs of incarceration or strangulation.

Laboratory investigations revealed a hemoglobin of $14.1 \mathrm{~g} / \mathrm{dl}$, an elevated white cell count $\left(13,400 / \mathrm{mm}^{3}\right.$ ), a normal renal profile (urea $23.2 \mathrm{mg} / \mathrm{dl}$; creatinine $1.1 \mathrm{mg} / \mathrm{dl}$ ) and a serum amylases of 57 IU. Abdominal X-ray showed diffuse dilatation of the small bowel with presence of pneumoperitoneum, and ultrasonography of the abdomen and pelvis revealed a thickening of small bowel loops, confirmed the presence of pneumoperitoneum and showed a moderate amount of free fluid diffusely spread in the peritoneal cavity. With a diagnosis of perforated acute abdomen an emergency laparotomy was performed. The abdominal cavity was opened by an upper midline incision and we found a moderate amount of fecal and purulent fluid in the cavity and the presence of multiple $\mathrm{JD}$, located mainly in the proximal $50 \mathrm{~cm}$ of the jejunum (fig. 1). One situated approximately $15 \mathrm{~cm}$ below the duodenal-jejunal flexure presented small perforation with 2 inches in diameter which intestinal contents drained. Through the wall of this perforated diverticulum it was possible to palpate a large enterolith $12 \mathrm{~cm}$ in major diameter. Initially, we chose to close the local perforation and to perform an extensive abdominal washout. We performed a resection of $50 \mathrm{~cm}$ of small bowel, $10 \mathrm{~cm}$ below the Treitz angle, removing the entire area containing JD. The intestinal transit was reconstituted by a single-layer end-to-end anastomosis with monofilament absorbable stitches. The umbilical hernia was corrected during closure of the abdominal wall without the need for a prosthetic mesh. The patient presented a favorable uncomplicated postoperative course and was discharged on the 5 th day. One year after surgery, the patient showed no more abdominal pain and had recovered her initial weight.

Macroscopic examination showed a segment of small intestine approximately $55 \mathrm{~cm}$ in length, with an outsider diameter ranging from 2.0 to $3.5 \mathrm{~cm}$, with reddish external serosa covered by fibrin plaques and purulent fluid. On the antimesenteric side the intestinal wall presented multiple JD with areas of thinning. Inside one of these diverticula we founded a large enterolith $12 \mathrm{~cm}$ in major diameter with an irregular yellowish outer surface (fig. 2 ) that occupied the interior of the diverticulum and protruded into the intestinal lumen, causing partial obstruction. This JD had a perforation approximately $2.0 \mathrm{~cm}$ in diameter, which connected the small bowel lumen with the peritoneal cavity.

Histological sections showed small bowel dilatation and wall thinning of the jejunal mucosal, areas of necrosis, hemorrhage and intense neutrophilic exudate that covered all the coats of the wall of the small bowel. The wall of the JD containing the enterolith showed a fistula in continuity with the hole drilled in the region of insertion of the intestinal mesentery. Biochemical analysis of the enterolith showed that it was composed of choleic acid, the end product of bile salt metabolism, and calcium oxalate.

\section{Discussion}

JD was first described in 1794 as a rarely found disease with an incidence rate ranging from 0.3 to $1.3 \%$ in autopsy series to $2.3 \%$ in radiographic findings [10]. Despite being a rare disease, there is a greater prevalence in older patients presenting with abdominal pain $[2,7]$. Of the patients with uncomplicated JD, the majority are asymptomatic or typically present with diffuse abdominal pain that radiates down the left side of the abdomen $[7,10]$. In symptomatic patients, flatulent dyspepsia (epigastric pain, abdominal discomfort, flatulence 1 or 2 hours after meals), epigastric cramping pain, bloating tenderness, fever and abdominal fullness are usually reported $[1,2,10]$. Anemia due to chronic occult gastrointestinal bleeding, iron or B12 deficiency has often been reported and commonly attributed to malabsorption syndrome, diarrhea and steatorrhea [11]. Malabsorption could be justified by the nonsynchronous peristaltic movement of the bowel, the dilation of the diverticula, the stasis of the intestinal content and the bacterial overgrowth $[1,11]$. 
Thirty percent of patients develop symptoms, and 10\% may present one of the associated complications that necessitate surgical intervention [7]. JD can be complicated not only by diverticulitis, but by hemorrhage, perforation, intussusception, volvulus, and even small bowel obstruction or perforation due to enteroliths formed inside these diverticula $[1-4,12]$. Complications of JD warranting surgical intervention occur in $10-30 \%$ of patients and the most common acute complications include diverticulitis, bleeding, enterolith formation, intestinal obstruction and perforation $[3,4,12]$. The incidence of jejunal diverticulitis with or without perforation ranges from 2 to $6 \%$ [13]. Intestinal subocclusion, reported in $10-25 \%$ of cases, is usually associated with JD as a result of peritonitis (following diverticulitis), perforation, strangulation and incarceration of an enterolith within a diverticulum [13]. Patients may present intestinal obstruction due to extruded enteroliths formed inside JD [1,7]. JD complicated by perforation or obstruction presented a high mortality rate in the past, however the mortality has been minimized because of the amelioration of diagnostics, antibiotic therapy and surgical protocols [13].

The pathogenesis of enteroliths within JD still remains controversial. Two theories have been proposed to explain the formation of enteroliths within JD [7, 12]. De novo stone formation theorized that enterolith is formed from choleic acid, the end product of bile salt metabolism. Deconjugation of bile salts by bacteria associated with the stasis of intestinal contents within the diverticulum, arising from the lack of peristalsis by the absence of muscular layer, predisposes them to precipitate and result in stone formation due to the acidic $\mathrm{pH}$ found in the proximal small bowel $[1,7,12,14]$. Another etiological basis for stone formation is stagnation of food particles due to abnormal transit with resultant debris accumulation and stone formation. These stones can then remain within the JD and eventually lead to various complications [1]. The presence of cholelithiasis is common in patients with enteroliths. However, the high incidence of cholelithiasis in the elderly population makes it difficult to establish a common origin for both conditions $[10,11]$.

JD is a challenging disorder from preoperative diagnostics. Plain abdominal radiography series of the abdomen are useful in the diagnosis of complications and may demonstrate evidence of perforation such as pneumoperitoneum or intestinal obstruction [15]. Occasionally, when the enterolith is rich in calcium oxalate, making it radiopaque, abdominal radiography series can identify it. The literature has shown that CT scan has variable reliability, while barium swallows are the gold standard for diagnosis [1]. CT may identify localized intestinal wall thickening due to inflammation or edema, abscesses, free abdominal fluids and pneumoperitoneum [16]. Multislice CT seems to be promising in diagnosing JD and appears more specific than enteroclysis concerning small bowel diseases and may identify the enterolith inside the JD or protruding into the intestinal lumen [16]. Endoscopic procedures, such as single- or double-balloon enteroscopy and wireless capsule endoscopy, are useful in diagnosing small bowel disorders, however these procedures are expensive, present low availability and cannot be used in the emergency setting, such as intestinal obstruction or perforation $[17,18]$. It has been shown that both procedures are not free of risk [18]. Double-balloon enteroscopy may be associated with an increased risk of jejunal perforation [17]. Wireless capsule endoscopy is a new hopeful technique for the detection of small bowel diseases and is predominantly used in cases of occult intestinal bleeding. However the presence of a large JD is a relative contraindication to capsule endoscopy because of the possibility of capsule entrapment in 
the diverticulum [18]. Thus, the use of this method in patients with JD should be chosen with relative prudence [18]. The laparoscopic approach can be very useful in investigating patients with a complicated symptomatology [19]. Laparoscopy becomes a valid diagnostic approach for complicated cases, it is rapidly convertible into laparotomy, can show the area of the intestinal complication and can guide the surgeon to the ideal incision site on the abdominal wall, minimizing postoperative pain and morbidity due to a larger abdominal incision [19].

The treatment of JD with perforated diverticulitis usually requires surgical indication. If the perforation of a JD causes only localized peritonitis without the presence of enteroliths and when the patient remains stable, it has been reported that a trial of nonsurgical management with intravenous antibiotics and other supportive measures alongside percutaneous CT-guided aspiration of localized intraperitoneal collections may be suitable and avoid the need for surgery [4]. However, the treatment of choice for perforated JD with presence of enteroliths causing generalized peritonitis is prompt segmental intestinal resection and primary anastomosis associated with careful washing of the peritoneal cavity $[1,10,11]$. The extent of the bowel resection depends on the length of the bowel affected by JD and the patient's operative condition [4]. If JD disease is extensive, the intestinal resection may be limited to include only the segment containing the perforated diverticula and to leave a segment of small bowel that still contains nonperforated diverticula, in order to avoid short bowel syndrome [4]. However, when the JD is located only in a small intestinal segment, as found in our patient, resection of the affected intestine can be achieved without loss of postoperative nutrition. Recently, the total laparoscopic approach has been used as a valid surgical strategy, even in cases of complicated diverticulitis [19]. When the patient's clinical condition makes surgical resection a high-risk procedure, some authors have proposed only the removal of enteroliths by enterotomy and debridement of the edges of JD with primary suture of the perforation, associated or not with drainage of the peritoneal cavity [2].

\section{Conclusion}

JD is typically a benign condition that affects particularly the elderly population. Sometimes the disease can lead to complications such as intestinal perforation or perforation due to enteroliths. A high degree of suspicion is necessary in view of the high mortality and morbidity rates resulting from a delayed diagnosis of the disease. When complications occur, surgical excision of the affected intestinal segment is the best therapeutic option.

\section{Acknowledgment}

C.M.G.S. thanks the Coordenação de Aperfeiçoamento de Pessoal de Nível Superior (Capes) for a Master Degree Scholarship. 


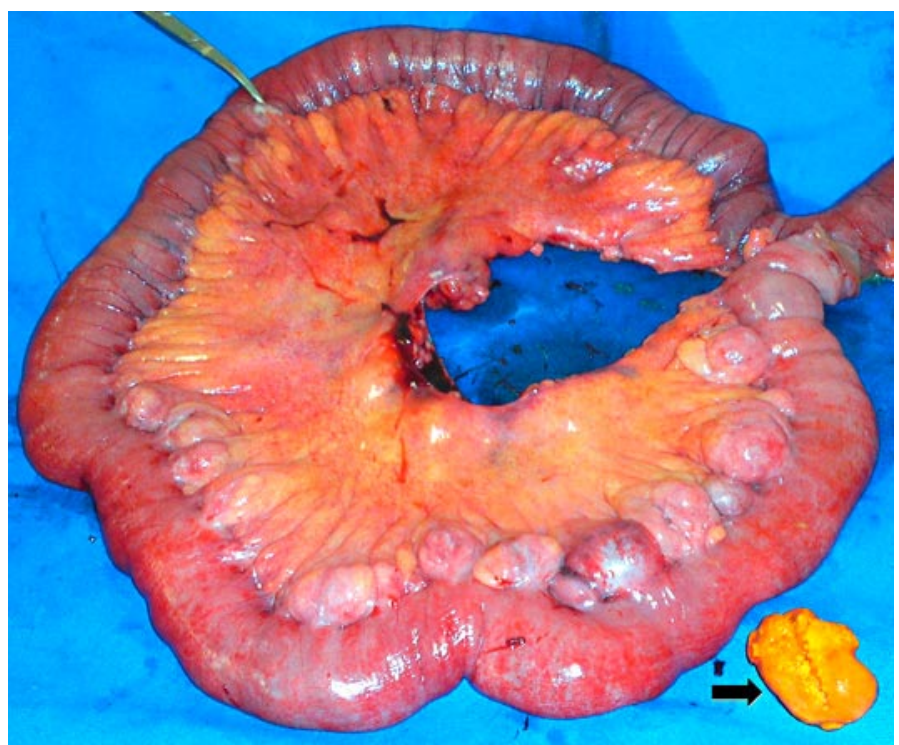

Fig. 1. Resected dilated loops of proximal jejunum with multiple diverticula and enterolith (arrow).

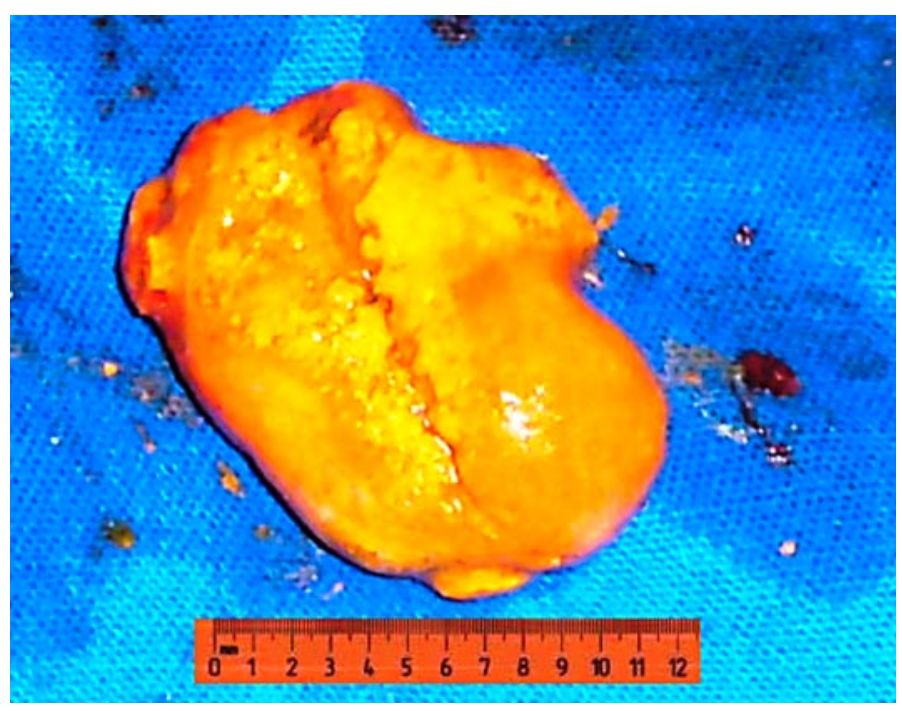

Fig. 2. External view of the enterolith measuring $12 \mathrm{~cm}$ in length found inside the jejunal diverticulum. 


\begin{tabular}{r|l|l|l}
$\begin{array}{r}\text { Case Reports in } \\
\text { Gastroenterology }\end{array}$ & $\begin{array}{l}\text { Case Rep Gastroenterol 2011;5:445-451 } \\
\text { DOl: 10.1159/000330842 }\end{array}$ & $\begin{array}{l}\text { Published online: } \\
\text { August 18, 2011 }\end{array}$ & $\begin{array}{l}\text { @ 2011 S. Karger AG, Basel } \\
\text { ISSN 1662-0631 } \\
\text { www.karger.com/crg }\end{array}$ \\
\hline
\end{tabular}

\section{References}

1 Chugay P, Choi J, Dong XD: Jejunal diverticular disease complicated by enteroliths: Report of two different presentations. World J Gastrointest Surg 2010;2:26-29.

$\longrightarrow 2$ Hayee B, Khan HN, Al-Mishlab T, McPartlin JF: A case of enterolith small bowel obstruction and jejunal diverticulosis. World J Gastroenterol 2003;9:883-884.

-3 Yaqub S, Evensen BV, Kjellevold K: Massive rectal bleeding from acquired jejunal diverticula. World J Emerg Surg 2011;6:17.

4 Butler JS, Collins CG, McEntee GP: Perforated jejunal diverticula: a case report. J Med Case Reports 2010;4:172.

5 Zager JS, Garbus JE, Shaw JP, Cohen MG, Garber SM: Jejunal diverticulosis: a rare entity with multiple presentations, a series of cases. Dig Surg 2000;17:643-645.

6 Staszewicz W, Christodoulou M, Proietti S, Demartines N: Acute ulcerative jejunal diverticulitis: Case report of an uncommon entity. World J Gastroenterol 2008;14:6265-6267.

7 Harris LM, Volpe CM, Doerr RJ: Small bowel obstruction secondary to enterolith impaction complicating jejunal diverticulitis. Am J Gastroenterol 1997;92:1538-1540.

8 Monchal T, Hornez E, Bourgouin S, Sbardella F, Baudoin Y, Butin C, Salle E, Thouhard H: Enterolith ileus due to jejunal diverticulosis. Am J Surg 2010;199:e45-e47.

-9 Patel VA, Jefferis H, Spiegelberg B, Iqbal Q, Prabhudesai A, Harris S: Jejunal diverticulosis is not always a silent spectator: A report of 4 cases and review of the literature. World J Gastroenterol 2008;14:5916-5919.

10 Kassahun WT, Fangmann J, Harms J, Bartels M, Hauss J: Complicated small-bowel diverticulosis: a case report and review of the literature. World J Gastroenterol 2007;13:2240-2242.

11 Balducci G, Dente M, Cosenza G, Mercantini P, Salvi PF: Multiple giant diverticula of the foregut causing upper gastrointestinal obstruction. World J Gastroenterol 2008;14:3259-3261.

12 Liu CH, Huang KW, Mo YH, Yang PM: Enterolith ileus in a patient with jejunal diverticulosis: sonographic findings. J Clin Ultrasound 2007;35:169-173.

13 Falidas E, Vlachos K, Mathioulakis S, Archontovasilis F, Villias C: Multiple giant diverticula of the jejunum causing intestinal obstruction: report of a case and review of the literature. World J Emerg Surg 2011;6:8.

14 Martinez CAR, Priolli DG, Palma RT, Waisberg J, Margarido NF: Intestinal obstruction due to enterolith formed in diverticulum of Meckel: case report. Rev Bras Coloproctol 2004;24:165-169.

15 Benya EC, Ghahremani GC, Brosnan JJ: Diverticulitis of the jejunum. Clinical and radiological features. Gastrointest Radiol 1991;16:24-28.

16 Bitterling H, Rock C, Reiser M: Computed tomography in the diagnosis of inflammatory bowel disease methodology of MSCT and clinical results. Radiologe 2003;43:17-25.

17 Hussain SA, Esposito SP, Rubin M: Identification of small bowel diverticula with double-balloon enteroscopy following non-diagnostic capsule endoscopy. Dig Dis Sci 2009;54:2296-2297.

18 Miehlke S, Tausche AK, Brückner S, Aust D, Morgner A, Madisch A: Retrieval of two retained endoscopy capsules with retrograde double-balloon enteroscopy in a patient with a history of complicated small-bowel disease. Endoscopy 2007;39(suppl 1):E157.

19 Garg N, Khullar R, Sharma A, Soni V, Baijal M, Chowbey P: Total laparoscopic management of large complicated jejunal diverticulum. J Minim Access Surg 2009;5:115-117. 